\title{
A direct approach towards the preparation of intermediates to the synthesis of Amaryllidaceae alkaloids from Morita-Baylis-Hillman adducts
}

Hamid Ullah and Fernando Coelho*

Laboratory of Synthesis of Natural Products and Drugs, Institute of Chemistry, State University of Campinas, UNICAMP, 13084-971, Campinas, SP, Brazil.

*Corresponding author. Tel.: (+55) 19-3521-3085; Fax: (+55) 19-3521-3023; e-mail: coelho@iqm.unicamp.br Keywords: Amaryllidaceae Alkaloids, Morita-Baylis-Hillman (MBH) Reaction, Stereoselective Synthesis.

\section{INTRODUCTION}

Plants from the Amaryllidaceae family are famous due to its pharmacological relevance. ${ }^{1}$ Many of these alkaloids have potent biological activities. The highly sophisticated substitution pattern of the carbon skeleton of these alkaloids, associated with their relevant biological and pharmacological significance, induced the interest of the organic synthesis community The goal of this work is the total synthesis of plicamine skeleton (7) and Narciclasin skeleton (8) (Figure 1) from MoritaBaylis-Hillman (MBH) adducts.

Figure 1. Carbon skeleton of Amaryllidaceae alkaloids
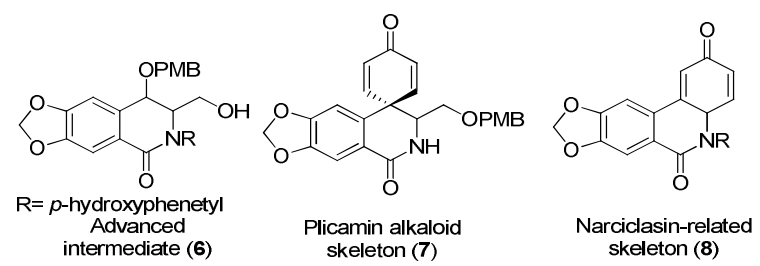

RESULTS AND DISCUSSION

Our approach begins with $\mathrm{MBH}$ adduct 2 which was obtained from aldehyde 1 in $53 \%$ and in $78 \%$ with ionic liquid (1-butyl-3-methylimidazolium bromide ([bmim]Br). ${ }^{2}$ Adduct 2 was therefore reduced to 3 in the presence of DIBAL- $\mathrm{H}^{2}$ to afford allylic diol 3 in $44 \%$ yield. The treatment of diol 3 with $p$-methoxy benzaldehyde gave the corresponding ketal 4 in $70 \%$ yield, after purification by neutral alumina column chromatography. ${ }^{3}$ Unfortunately attempts to ozonolyse the double bond of $\mathbf{4}$ failed. $^{4}$ To circumvent this issue we increase the reaction time but we were able to isolate only overoxidation products (Scheme 1). Because of this result, we change the synthetic sequence. Then, an ozonolysis reaction was carried out directly on allylic diol 3 to afford keto-diol 9 in 70\% yield (Scheme 2).

Compound $\mathbf{9}$ will be used for further chemical transformations. Thus, the hydroxyl groups will be protected as the corresponding 1,3-dioxanyl.

The carbonyl group of $\mathbf{9}$ will be used as substrate for a reductive amination reaction with benzyl- or phenethylamine to obtain 10, which in turn will be used toward the synthesis of 6 (Scheme 2).

\section{Scheme1.}

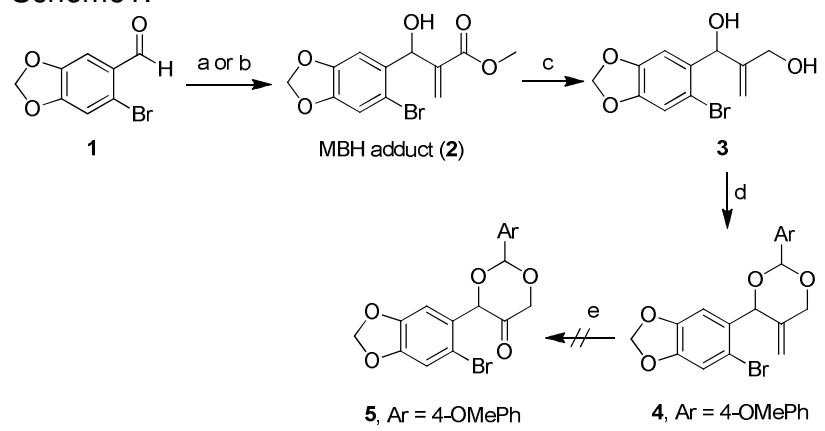

Reagents and conditions: a) Methyl acrilate, $\mathrm{DABCO}$, r.t., 38 days, $53 \%$ or b) Methyl acrilate, DABCO, [bmim]Br, r.t., 5 days, $78 \%$. c) DIBAL-H (2.0 eq.), $\mathrm{CH}_{2} \mathrm{Cl}_{2},-78^{\circ} \mathrm{C}, 1 \mathrm{~h}$, $44 \%$. d) $p$-anisaldehyde, CSA, $25 \square \mathrm{C}, 13 \mathrm{~h}, 70 \%$ e) $\mathrm{O}_{3}$, $\mathrm{MeOH} / \mathrm{CH}_{2} \mathrm{Cl}_{2}(1: 4),-72{ }^{\circ} \mathrm{C} ; 10$ eq. $\mathrm{S}\left(\mathrm{CH}_{3}\right)_{2},-72{ }^{\circ} \mathrm{C}$, 3hr.

Scheme 2.

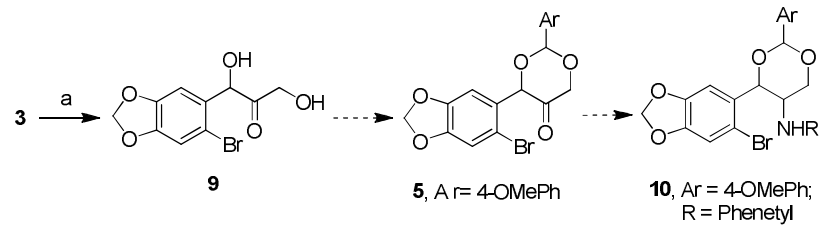

Reagents and conditions: a) $\mathrm{O}_{3}, \mathrm{MeOH} / \mathrm{CH}_{2} \mathrm{Cl}_{2}$ (1 : 4), $-72{ }^{\circ} \mathrm{C} ; 10$ eq. $\mathrm{S}\left(\mathrm{CH}_{3}\right)_{2},-72{ }^{\circ} \mathrm{C}, 3 \mathrm{~h}, 70 \%$.

\section{CONCLUSION}

The synthesis of $\mathbf{5}$ is described in 3 steps from $\mathrm{MBH}$ adduct 2. This intermediate should allow the access to an advanced compound to be used in the synthesis of Amaryllidaceae alkaloids.

\section{ACKNOWLEDGEMENTS}

We thank CNPq and FAPESP for financial support.

\section{REFERENCES}

1 Petit, G.R.; Gaddamidi, V.; Herald, D.L.; Singh, S.B.; Cragg, G.M.; Schmidt, J.M.; Boettner, F.E.; Williams, M.; Sagawa, Y. J. Nat. Prod. 1986, 49, 995;

${ }^{2}$ Coelho, F.; Lopes, E.C.S. J. Braz. Chem. Soc. 2007, 18, 1415.

${ }^{3}$ M.Smith,D.H.; Rammler.D.H.; Goldberg,I.H and khorana, H.G. J. Am. Chem. Soc. 1984, 430, 1962.

${ }^{4}$ Coelho, F.; Abella, C.A.M.; Rezende, P.; Souza, M. L. Tetrahedron Lett. 2008, 49, 145. 